\title{
SECOND-ORDER PERTURBATIONS IN ALFVÉN WAVES IN COLD PLASMA APPROXIMATION
}

\author{
I.S. Dmitrienko \\ Institute of Solar-Terrestrial Physics SB RAS, \\ Irkutsk, Russia, dmitrien@iszf.irk.ru
}

\begin{abstract}
The second-order amplitude perturbations driven by Alfvén waves are studied. Equations for such second-order perturbations are derived and their solutions are found. The second-order perturbations are shown to be generated by the magnetic pressure of the waves. They represent plasma flows and magnetic field perturbations in a plane perpendicular to the direction of the field perturbation and plasma displacement in the Alfvén wave. In connection with the interpretation of fast plasma flows observed in the magnetotail, of particular interest is the description of second-order flows, which relates their properties to properties of Alfvén
\end{abstract}

waves and the disturbance that generates these waves. The results suggest that at least some of the fast plasma flows observed in the magnetotail can be one of the manifestations of propagating Alfvén waves. The environment model and cold plasma approximation in use are quite applicable for the plasma sheet boundary layers, where an essential part of the fast plasma flows occurs. flows.

Keywords: Alfvén waves, magnetotail, fast plasma

\section{INTRODUCTION}

The study of second-order perturbations in Alfvén waves is of interest primarily in connection with the interpretation of observed plasma flows in the magnetotail [Zong et al., 2007; Fruhauff, Glassmeier, 2016]. Alfvén waves propagating both to and from Earth have been long and widely observed in the magnetotail [Keiling et al., 2000, 2005; Takada et al., 2005, 2006; Keiling, 2009]. A source of Alfvén perturbations in the tail lobes and adjacent plasma sheet boundary layer (PSBL) can be perturbations penetrating from the solar wind, and instabilities of different types, including reconnection in the magnetotail [Lee, 1998; Leonovich et al., 2003; Walker, 2005; Klimushkin et al., 2012; Leonovich, Kozlov, 2013; Mazur, Chuiko, 2013; Birn et al., 2015; Mager et al., 2017]. Alfvén perturbations can also arise due to transformation of fast magnetosonic modes (FMS modes) of the waveguide in the magnetotail [Wright, Allan, 2008; Mazur et al., 2010; Dmitrienko, 2013]. At the same time, in this region there are so-called fast plasma flows propagating, like Alfvén waves, to and from Earth, depending on the point of observation. Along with these flows, magnetic perturbations are usually recorded [Cao et al., 2006; Zong et al., 2007; Du et al., 2011; Fruhauff, Glassmeier, 2016].

The formation of the flow in Alfvén waves has been explored when studying nonlinear effects in Alfvén waves [Dmitrienko, 1997]. Dmitrienko [2011] considered a monochromatic wave with the time envelope arising from FMS-wave transformation as a source of formation of such a flow. The flow obtained in this model is not limited to propagation direction, as well as the wave that generates it. Such a pattern of the wave and its associated flow is inconsistent with the observed perturbations whose size in the direction along the geomagnetic field is finite.
This paper examines the Alfvén waves generated by the initial perturbation with finite size in two coordinates, one of which is directed along the unperturbed magnetic field. The Alfvén waves oppositely propagating along the unperturbed magnetic field, which are driven by this initial perturbation, have a finite size in the direction of their propagation. This formulation of the problem allows us to trace propagation of Alfvén waves to and from Earth and the evolution of their driven second-order perturbations, including plasma flows associated with these waves. The choice of the fairly simple model of medium is explained by the fact that it can analytically describe the formation of the secondorder perturbations and relate the characteristics of the fast plasma flows in Alfvén waves to the characteristics of the perturbation that generates these waves. This model does not, of course, reflect the structure of the geomagnetic tail, in which Alfvén perturbations are presumably formed, but it can adequately describe the phenomenon under study. A very large number of observations of fast plasma flows are made in PSBL, where the cold plasma approximation is quite valid (the $\beta$ parameter varies in the region from 0.25 near the plasma sheet to $\beta<0.1$ in tail lobes).

The strong magnetic field toward Earth or from it (depending on from which of the two tail lobes the boundary is examined) ensures that the effect of the small magnetic field component across the tail is weak. This allows us to consider the magnetic field in PSBL as direct. An Alfvén perturbation propagates along the field, therefore it and its associated second-order perturbation do not go beyond the range of applicability of the approximation in use. The only essential feature of PSBL, which is ignored in the model of medium, is the Alfvén velocity inhomogeneity in a direction perpendicular both to the field direction and to the azimuth direction. The effect of this factor on the results is discussed in Conclusion. 
It should be noted that there is a direction in the study of the formation of flows in Alfvén waves as a result of the development of the secondary instability, caused by nonlinear effects. This direction is, in particular, represented in [Pokhotelov et al., 2003, 2004; Zhao et al., 2012]. These papers have mentioned the fundamental role of kinetic effects in generating convective motions. In the present paper, the formulation of the problem is different, which allows us to obtain secondorder flows generated by nonlinear effects in the cold plasma approximation. This is due to the difference in problem setting, which is explained by focusing on different physical phenomena. The papers [Pokhotelov et al., 2003, 2004; Zhao et al., 2012] aim at applying the results to stationary turbulent processes, while the present work focuses on its application to perturbations localized in space and time. Accordingly, in [Pokhotelov et al., 2003, 2004; Zhao et al., 2012] convection flows are identified, if it is formulated in terms of harmonic decomposition as a zero longitudinalcoordinate harmonic, by corresponding averaging. In this case, the averaged longitudinal ponderomotive force exists only due to small effects - dissipative [Dmitrienko, 1997] or kinetic [Pokhotelov et al., 2003, 2004; Zhao et al., 2012]. In this paper, we examine the longitudinally localized Alfvén perturbations and correspondingly the second-order flows, which are also longitudinally localized. The longitudinal ponderomotive force generating such flows exists in the absence of small effects too. In addition, in [Pokhotelov et al., 2003, 2004; Zhao et al., 2012], the solution is found only as a perturbation of small transversal scales; for the perturbation considered in the present work from a certain initial time, no assumptions are made about its transversal scale. When the transversal scale of Alfvén perturbation tends to zero, the transversal component of the velocity of the flow obtained in this paper disappears in agreement with the fact that [Pokhotelov et al., 2003, 2004; Zhao et al., 2012] convection flows for the transversely-small-scale Alfvén waves are produced only by kinetic effects. The transversal scale has no effect on the existence of the longitudinal flow.

\section{INITIAL EQUATIONS}

As initial equations we use

$$
\begin{gathered}
\partial_{t} \mathbf{B}=\nabla \times(\mathbf{v} \times \mathbf{B}), \\
\rho d_{t} \mathbf{v}=\frac{1}{4 \pi}(\nabla \times \mathbf{B}) \times \mathbf{B}, \\
\partial_{t} \rho+\nabla \cdot \rho \mathbf{v}=0 .
\end{gathered}
$$

Here $\mathbf{B}$ is the magnetic field, $\rho$ is the plasma density, $\mathbf{v}$ is the plasma velocity. We believe that when unperturbed the plasma is at rest in a uniform magnetic field $\mathbf{B}_{0} ; \rho_{0}$ is the unperturbed uniform plasma density. Given the presence of perturbation, replace notations as follows $\mathbf{B} \rightarrow \mathbf{B}_{0}+\mathbf{B}, \rho \rightarrow \rho_{0}+\rho$, where $\mathbf{B}$ and $\rho$ are the field and density perturbations; $\mathbf{v}$ is the plasma velocity in the perturbation. Direct the Z-axis of the Cartesian coordinate system along $\mathbf{B}_{0}$.

Suppose that in plasma there is an initial perturbation uniform in a direction $y$, which is specified by a function of $z$ and $x$. Hence, the resulting perturbation is a function of $z$ and $x$. Assume that in this perturbation the $x$ and $z$ field and velocity components as well as density are small, of order of $\varepsilon, \varepsilon<<1$ :

$$
\frac{B_{x}}{B_{0}} \sim \frac{B_{z}}{B_{0}} \sim \frac{v_{x}}{V_{a}} \sim \frac{v_{z}}{V_{a}} \sim \frac{\rho}{\rho_{0}} \sim \varepsilon, \varepsilon<<1,
$$

$V_{a}$ is the Alfvén velocity:

$$
V_{a}=\frac{B_{0}}{\sqrt{4 \pi \rho_{0}}} \text {. }
$$

Impose no restrictions on $B_{y}$ and $v_{y}$, assume only that they are of the same order:

$$
\frac{v_{y}}{V_{a}} \sim \frac{B_{y}}{B_{0}} .
$$

Dropping the terms in (1) that, according to (2), (3), are negligible compared to the remaining ones, obtain the equations

$$
\begin{aligned}
\partial_{t} b_{y} & =\partial_{z} v_{y}, \partial_{t} v_{y}=V_{a}^{2} \partial_{z} b_{y} ; \\
\partial_{t} b_{x}= & \partial_{z} v_{x} ; \\
& \partial_{t} b_{z}=-\partial_{x} v_{x} ; \\
& \partial_{t} v_{x}=V_{a}^{2} \partial_{z} b_{x}-V_{a}^{2} \partial_{x} b_{z}-V_{a}^{2} \partial_{x} b_{y}^{2} / 2 ; \\
& \partial_{t} v_{z}=-V_{a}^{2} \partial_{z} b_{y}^{2} / 2 ; \\
& \partial_{t} \rho=-\rho_{0}\left(\partial_{x} v_{x}+\partial_{z} v_{z}\right) .
\end{aligned}
$$

We designate $\mathbf{b}=\mathbf{B} / \boldsymbol{B}_{0}$. Equations (4) are for Alfvén waves, and system (5)-(9) includes equations for the $x$ and $z$ field and velocity components as well as for density. System (5)-(9) includes the magnetic pressure proportional to $b_{y}^{2}$. It is produced by Alfvén waves, for which (4) yields the equation

$$
\partial_{t t} b_{y}=V_{a}^{2} \partial_{z z} b_{y} \text {. }
$$

In system (5)-(9), the Alfvén wave magnetic pressure serves as a driver generating the perturbation $b_{x}, b_{z}$, $v_{x}, v_{z}, \rho$. Our purpose is to describe these perturbations. Note that to derive (4), (5)-(9) does not require any restrictions on the amplitude of Alfvén waves, i.e. directly on $b_{y}$ and $v_{y}$. However, since the perturbations described by (5)-(9) are generated by the magnetic pressure of Alfvén waves, their amplitude is determined by the magnetic pressure $b_{y}^{2}$. For condition (2) to hold, the condition $B_{y} \sim \varepsilon^{1 / 2}$ should be fulfilled. This condition is sufficiently weak, which is essential for the application of the results to specific magnetospheric phenomena.

\section{SECOND-ORDER PERTURBATIONS}

Concretize $b_{y}$ according to (10). Suppose that there is an initial plasma displacement at which the velocity at the initial time $v_{y}(x, z, 0)=W(x, z)$, then $\partial_{t} b_{y}(x, z, 0)=$ $=\partial_{z} W(x, z) \neq 0$; there is no field perturbation at the initial time. The nondimensionalized perturbation of the magnetic field component $y$ in the Alfvén waves, generated by the initial perturbation $W(x, z)$, is 


$$
\begin{aligned}
& b_{y}(x, z, t)=b_{y+}+b_{y-}, \\
& b_{y+}=-b\left(x, z+V_{a} t\right), \\
& b_{y-}=b\left(x, z-V_{a} t\right),
\end{aligned}
$$

where $b(x, z)=-W(x, z) /\left(2 V_{a}\right)$. It comprises two Alfvén waves propagating along $z$ in opposite directions from the initial perturbation. The magnetic pressure caused by the Alfven waves is

$$
b_{y}^{2}(x, z, t)=b_{y \pm}^{2}(x, z, t)+\bar{b}_{y}^{2}(x, z, t),
$$

where

$$
\begin{aligned}
& b_{y \pm}^{2}(x, z, t)=b_{y+}^{2}+b_{y-}^{2}, \\
& b_{y+}=b^{2}\left(x, z+V_{a} t\right), \\
& b_{y-}=b^{2}\left(x, z-V_{a} t\right)
\end{aligned}
$$

and

$$
\bar{b}_{y}^{2}(x, z, t)=-2 b\left(z-V_{a} t\right) b\left(z+V_{a} t\right) .
$$

It is clear that $b_{y \pm}^{2}(x, z, t)$ is the magnetic pressure in the Alfvén waves propagating in opposite directions along the $\mathrm{Z}$-axis from the initial perturbation, $\bar{b}_{y}^{2}(x, z, t)$ is the magnetic pressure perturbation in the vicinity of the initial perturbation. Assume that the initial perturbation exists in the finite region of the $\mathrm{XZ}$ plane. In this case, we can hold that there is a finite time during which the plasma perturbation regions in each oppositely propagating Alfvén wave intersect.

To solve (5)-(9), specify zero initial conditions such that $t=0 \quad b_{x}=b_{z}=0, v_{x}=v_{z}=0, \rho=0$. From (5)-(7) it is easy to derive the equation for $v_{x}$

$$
\left(\partial_{t t}-V_{a}^{2} \Delta\right) v_{x}=-\frac{1}{2} V_{a}^{2} \partial_{t} \partial_{x} b_{y}^{2}
$$

with $\Delta=\partial_{x x}+\partial_{z z}$. This equation is further substituted for equation (7) in system (5)-(9).

Note that (8), (14) can also yield an equation for $\nabla \cdot \mathbf{v}=\partial_{x} v_{x}+\partial_{z} v_{z}$

$$
\left(\partial_{t t}-V_{a}^{2} \Delta\right) \partial_{t} \nabla \cdot \mathbf{v}=-\frac{1}{2} V_{a}^{2}\left(\partial_{t t}-V_{a}^{2} \Delta\right) \Delta b_{y}^{2},
$$

from which it follows that a propagating Alfvén wave can produce only an incompressible flow.

For the longitudinal plasma velocity component we have equation (8). Use notations $v_{z+}$ and $v_{z-}$ for the longitudinal velocity perturbations generated by individual Alfvén waves with magnetic pressure $b_{y+}^{2}$ and $b_{y-}^{2}$; denote also

$$
v_{z \pm}=v_{z+}+v_{z-} \text {. }
$$

Obviously, from (8) we get

$$
\partial_{t} v_{z \pm}=-\frac{1}{2} V_{a}^{2} \partial_{z} b_{y \pm}^{2}(x, z, t),
$$

which yields

$$
v_{z+}=-\frac{1}{2} V_{a} b^{2}\left(x, z+V_{a} t\right), v_{z-}=\frac{1}{2} V_{a} b^{2}\left(x, z-V_{a} t\right) \text {. }
$$

We can see that in each of the propagating waves plasma moves in the direction of wave propagation.
This movement is caused not by the initial perturbation, but directly by the pressure of propagating waves and remains unchanged when $t \rightarrow \infty$ in the form given by (16). Note that the formulas for longitudinal plasma velocity in Alfvén wave similar to (16) have been obtained in [Dmitrienko, 2011] for the Alfvén monochromatic wave with envelope, excited due to FMS-wave transformation in the transversely inhomogeneous plasma. For the $x$ plasma velocity component we have equation (14) with zero initial conditions. Represent $v_{x}$ as $v_{x}=v_{x \pm}+\bar{v}_{x}$, where

$$
\left(\partial_{t t}-V_{a}^{2} \Delta\right) v_{x \pm}=-\frac{1}{2} V_{a}^{2} \partial_{x} \partial_{t} b_{y \pm}^{2}
$$

are the initial conditions

$$
\begin{aligned}
& v_{x \pm}(x, z, 0)=0, \partial_{t} v_{x \pm}(x, z, 0)=0 ; \\
& \left(\partial_{t t}-V_{a}^{2} \Delta\right) \bar{v}_{x}=-\frac{1}{2} V_{a}^{2} \partial_{t} \partial_{x} \bar{b}_{y}^{2} ;
\end{aligned}
$$

are the initial conditions

$$
\bar{v}_{x}(x, z, 0)=0, \partial_{t} \bar{v}_{x}(x, z, 0)=0 .
$$

The perturbation $\bar{v}_{x}$ is not further considered because it decreases as it propagates due to the fact that its driver on the right side of the equation for $\bar{v}_{x}$ is localized in a certain region in the $\mathrm{XZ}$ plane and the finite time operates. As for $V_{x \pm}$, represent the solution of equation (17) as $v_{x \pm}=V_{x \pm}+V_{x}^{0}$, where $V_{x \pm}$ meets the conditions $\left(\partial_{t t}-V_{\alpha}^{2} \Delta\right) V_{x \pm}=0$ and $\partial_{x} V_{x \pm}=\frac{1}{2} \partial_{t} b_{y \pm}^{2}$. Obviously, if $V_{x \pm}$ satisfies the two conditions, $V_{x \pm}$ is a solution of (17). The solution $V_{x \pm}$ can be chosen as

$$
V_{x \pm}=\frac{V_{a}}{2} \int_{x_{0}}^{x} \partial_{z}\left(b^{2}\left(x^{\prime}, z+V_{a} t\right)-b^{2}\left(x^{\prime}, z-V_{a} t\right)\right),
$$

where $x_{0}$ is the arbitrary point. The solution of (18) does not satisfy the initial conditions of equation (17) since at $t=0 \quad \partial_{t} V_{x \pm} \neq 0$. We have

$$
\begin{aligned}
& \partial_{t} V_{x \pm}\left(x, z, 0, x_{0}\right)=u\left(x, z, x_{0}\right), \\
& u\left(x, z, x_{0}\right)=V_{a}^{2} \partial_{z z} \int_{x_{0}}^{x} b^{2}\left(x^{\prime}, z\right) d x^{\prime} .
\end{aligned}
$$

Denote also

$$
\begin{aligned}
& u^{+}\left(z, x_{0}\right)=V_{a}^{2} \partial_{z z} \int_{x_{0}}^{\infty} b^{2}\left(x^{\prime}, z\right) d x^{\prime}, \\
& u^{-}\left(z, x_{0}\right)=V_{a}^{2} \partial_{z z} \int_{x_{0}}^{-\infty} b^{2}\left(x^{\prime}, z\right) d x^{\prime} .
\end{aligned}
$$

Let the initial perturbation region be characterized in the $x$ and $z$ coordinates by the $L_{x}, L_{z}$, parameters, i.e. we can suppose $b^{2}(x, z)=0$ for $|x|>L_{x}$ and $|z|>L_{z}$. Generally speaking, $L_{x}$ and $L_{z}$ can be functions of $z$ and $x$ respectively, but to reduce the formulas we assume $L_{x}=\max \left|L_{x}(z)\right|, L_{z}=\max \left|L_{z}(x)\right|$. Then we have for $x>L_{x} \quad u\left(x, z, x_{0}\right)=u^{+}\left(z, x_{0}\right), \quad$ and for $x<-L_{x} u\left(x, z, x_{0}\right)=u^{-}\left(z, x_{0}\right)$. Notice that, although $V_{x \pm}(x, z, 0)=0$, the solution $V_{x \pm}(x, z, t) \neq 0$ for any $t \neq 0$ when $|x|=\infty$, i.e. infinitely far away from the initial 
perturbation. $V_{x \pm}$ can not therefore be regarded as a separate perturbation part having the physical meaning. Hence, find $v_{x \pm}$ - a complete solution of (17) with zero initial conditions. This requires the solution of the equation

$$
\left(\partial_{t t}-V_{a}^{2} \Delta\right) V_{x}^{0}\left(x, z, t, x_{0}\right)=0
$$

with the initial conditions $V_{x}^{0}\left(x, z, 0, x_{0}\right)=0$, $\partial_{t} V_{x}^{0}\left(x, z, 0, x_{0}\right)=-u$. Then for $v_{x \pm}=V_{x \pm}+V_{x}^{0}$ zero initial conditions will hold. The solution of (21) has the form

$$
\begin{aligned}
& V_{x}^{0}=-\frac{1}{2 \pi V_{a}} \times \\
& \times \int_{z-V_{a} t}^{z+V_{a} t} \int_{x-\sqrt{V_{a}^{2} t^{2}-\left(z-z^{\prime}\right)^{2}}}^{x+\sqrt{V^{2} t^{2}-\left(z-z^{\prime}\right)^{2}}} \frac{u\left(x^{\prime}, z^{\prime}, x_{0}\right) d x^{\prime} d z^{\prime}}{\sqrt{V_{a}^{2} t^{2}-\left(x-x^{\prime}\right)^{2}-\left(z-z^{\prime}\right)^{2}}} .
\end{aligned}
$$

Consider the solution $V_{x}^{0}$ for $|x| \rightarrow \infty$. Given $|x|>L_{x}+\sqrt{\left(V_{a} t+L_{z}\right)^{2}-z^{2}}$, the $x^{\prime}$ integration in (22) lies outside $\left(-L_{x}, L_{x}\right)$. So, for such $x$ we have $V_{x}^{0}=V_{x}^{0(+)}$,

$$
\begin{aligned}
& V_{x}^{0(+)}=-\frac{V_{a}}{2} \times \\
& \times \partial_{z} \int_{x_{0}}^{\infty}\left(b^{2}\left(x^{\prime}, z+V_{a} t\right)-b^{2}\left(x^{\prime}, z-V_{a} t\right)\right) d x^{\prime} \\
& \text { at } x>L_{x}+\sqrt{\left(V_{a} t+L_{z}\right)^{2}-z^{2}} \text { and } \\
& V_{x}^{0(-)}=-\frac{V_{a}}{2} \times \\
& \times \partial_{z} \int_{x_{0}}^{-\infty}\left(b^{2}\left(x^{\prime}, z+V_{a} t\right)-b^{2}\left(x^{\prime}, z-V_{a} t\right)\right) d x^{\prime} \\
& \text { at } x<-L_{x}-\sqrt{\left(V_{a} t+L_{z}\right)^{2}-z^{2}} .
\end{aligned}
$$

Thus, taking into account (18), we have $V_{x}^{0}=-V_{x \pm}$, hence $v_{x \pm}=0$ in (22), i.e. for sufficiently large $|x|$. Further, $V_{x}^{0}$ satisfies, in addition to (21), the equation

$$
\left(\partial_{t t}-V_{a}^{2} \Delta\right) \partial_{x} V_{x}^{0}=0
$$

with the initial conditions

$$
\begin{aligned}
& \partial_{x} V_{x}^{0}(x, z, 0)=0, \\
& \partial_{t} \partial_{x} V_{x}^{0}(x, z, 0)=V_{a}^{2} \partial_{z z} b^{2}(x, z) .
\end{aligned}
$$

Since $b^{2}(x, z)$ is localized in the $\mathrm{XZ}$ plane, the solution of (25) is not the wave propagating only along the Z-axis. Consequently, the amplitude of the perturbation corresponding to the solution of (25) decreases as the perturbation propagates from the source: the region, where $\partial_{x} V_{x}^{0} \neq 0$, increases, but $\partial_{x} V_{x}^{0} \rightarrow 0$ as the $V_{x}^{0}$ perturbation propagates (unlike the problem for $V_{x}^{0}$, in which the initial conditions do not tend to zero when $|x|$ $\rightarrow \infty$ ). The solution of this equation has a front whose shape tends to a circle $x^{2}+z^{2}=V_{a}^{2} t^{2}$ when $t \rightarrow \infty$; outside this circle the perturbation is absent; (22) gives an estimate of the position of the front of $\partial_{x} V_{x}^{0}$ at finite values of $t$, and (23), (24) yields $V_{x}^{0}$ where $\partial_{x} V_{x}^{0}=0$. The fact that $\partial_{x} V_{x}^{0}$ tends to zero in the front and behind it when $t \rightarrow \infty$ means that $V_{x}^{0}$ tends to the function independent of $x$. We denote this function as

$$
\left(V_{x}^{0}\right)_{a s}:\left(V_{x}^{0}\left(z, t, x_{0}\right)\right)_{a s}=\lim _{t \rightarrow \infty} V_{x}^{0}\left(x, z, t, x_{0}\right) .
$$

Find $\left(V_{x}^{0}\right)_{a s}$. Using that with $|x|>L_{x}$ for $u\left(x, z, x_{0}\right)$ (19), (20) are applicable, and the contribution of the $|x|<L_{x}$ integral to the inner integral on the right side of (22) tends to 0 as $t \rightarrow \infty$, we can write

$$
\begin{aligned}
& \left(V_{x}^{0}\right)_{a s}=-\frac{1}{2 \pi V_{a}} \times \\
& \times \int_{z-V_{a}^{t}}^{z+V_{a}^{t}} u^{+}\left(z^{\prime}, x_{0}\right) \int_{0}^{\sqrt{V_{a}^{2} t^{2}-\left(z-z^{\prime}\right)^{2}}} \frac{d \xi d z^{\prime}}{\sqrt{V_{a}^{2} t^{2}-\xi^{2}-\left(z-z^{\prime}\right)^{2}}}- \\
& -\frac{1}{2 \pi V_{a}} \times \\
& \times \int_{z-V_{a} t}^{z+V_{a} t} u^{-}\left(z^{\prime}, x_{0}\right) \int_{-\sqrt{V_{a}^{2} t^{2}-\left(z-z^{\prime}\right)^{2}}}^{0} \frac{d \xi d z^{\prime}}{\sqrt{V_{a}^{2} t^{2}-\xi^{2}-\left(z-z^{\prime}\right)^{2}}}
\end{aligned}
$$

where $\xi=x^{\prime}-x$. Thus,

$$
\left(V_{x}^{0}\right)_{a s}=-\frac{1}{4 V_{a}} \int_{z-V_{a} t}^{z+V_{a} t}\left(u^{+}\left(z^{\prime}, x_{0}\right)+u^{-}\left(z^{\prime}, x_{0}\right)\right) d z^{\prime} .
$$

Taking into account (19), (20), obtain that for $t \rightarrow \infty$

$$
\begin{aligned}
& \left(V_{x}^{0}\right)_{a s}=-\frac{V_{a}}{4} \partial_{z} \times \\
& \times \int_{x_{0}}^{\infty}\left(b^{2}\left(x^{\prime}, z+V_{a} t\right)-b^{2}\left(x^{\prime}, z-V_{a} t\right)\right) d x^{\prime}- \\
& -\frac{V_{a}}{4} \partial_{z} \int_{x_{0}}^{-\infty}\left(b^{2}\left(x^{\prime}, z+V_{a} t\right)-b^{2}\left(x^{\prime}, z-V_{a} t\right)\right) d x^{\prime} .
\end{aligned}
$$

Turning back to $V_{x \pm}$ as a particular solution of (17), make use of the fact that its choice has an arbitrariness, which allows us to choose a particular solution of (17) in the form of $\tilde{v}_{x \pm}$ :

$$
\tilde{v}_{x \pm}=V_{x \pm}+C_{1}\left(z+V_{a} t\right)+C_{2}\left(z-V_{a} t\right),
$$

where $C_{1}$ and $C_{2}$ are arbitrary functions of their arguments. We put

$$
C_{1}\left(z+V_{a} t\right)+C_{2}\left(z-V_{a} t\right)=\left(V_{x}^{0}\right)_{a s} .
$$

Then we get $\tilde{v}_{x \pm}$ in the form

$$
\tilde{v}_{x \pm}=\tilde{v}_{x+}+\tilde{v}_{x-},
$$

where

$$
\begin{aligned}
& \tilde{v}_{x+}(x, z, t)=\frac{V_{a}}{4} \times \\
& \times \partial_{z}\left(\int_{-\infty}^{x} b^{2}\left(x^{\prime}, z+V_{a} t\right) d x^{\prime}+\int_{\infty}^{x} b^{2}\left(x^{\prime}, z+V_{a} t\right) d x^{\prime}\right), \\
& \tilde{v}_{x-}(x, z, t)=-\frac{V_{a}}{4} \times \\
& \times \partial_{z}\left(\int_{-\infty}^{x} b^{2}\left(x^{\prime}, z-V_{a} t\right) d x^{\prime}+\int_{\infty}^{x} b^{2}\left(x^{\prime}, z-V_{a} t\right) d x^{\prime}\right) .
\end{aligned}
$$


As we can see, the function $\tilde{v}_{x \pm}$ is antisymmetric for large values of $|x|$ :

$$
\begin{aligned}
& \lim _{x \rightarrow \infty} \tilde{v}_{x+}(x, z, t)=-\lim _{x \rightarrow-\infty} \tilde{v}_{x+}(x, z, t)= \\
& =\frac{V_{a}}{4} \partial_{z}\left(\int_{-\infty}^{\infty} b^{2}\left(x^{\prime}, z+V_{a} t\right) d x^{\prime}\right), \\
& \lim _{x \rightarrow \infty} \tilde{v}_{x-}(x, z, t)=-\lim _{x \rightarrow-\infty} \tilde{v}_{x-}(x, z, t)= \\
& =\frac{V_{a}}{4} \partial_{z}\left(\int_{-\infty}^{\infty} b^{2}\left(x^{\prime}, z-V_{a} t\right) d x^{\prime}\right) .
\end{aligned}
$$

We have at the initial time

$$
\begin{aligned}
& \tilde{v}_{x \pm}(x, z, 0)=0, \\
& \partial_{t} \tilde{v}_{x \pm}(x, z, 0)=\frac{V_{a}^{2}}{2} \times \\
& \times \partial_{z z}\left(\int_{\infty}^{x} b^{2}\left(x^{\prime}, z\right) d x^{\prime}+\int_{-\infty}^{x} b^{2}\left(x^{\prime}, z\right) d x^{\prime}\right) .
\end{aligned}
$$

If we introduce a notation $\tilde{u}(x, z)$ for $\partial_{t} \tilde{v}_{x \pm}(x, z, 0): \tilde{u}(x, z)=\partial_{t} \tilde{v}_{x \pm}(x, z, 0)$ and then find a solution of the equation in the form of (21) for $V_{x}^{0}$, this solution has the form of (22), but with $u$ substituted for $\tilde{u}$. Thus, we obtain for $v_{x \pm}$ the representation $v_{x \pm}=\tilde{v}_{x \pm}+V_{x}^{0}$. Since $V_{x}^{0} \rightarrow 0$ at $t \rightarrow \infty$, then $v_{x \pm} \rightarrow \tilde{v}_{x \pm}$ at $t \rightarrow \infty$; $\tilde{v}_{x \pm}$ is given by (26)-(28). The function $\tilde{v}_{x \pm}$ is not zero in bands

$$
-\infty<x<\infty,-L_{z} \pm V_{a} t<|z|<L_{z} \pm V_{a} t .
$$

For large but finite $t$, ranges of non-zero $v_{x \pm}$ are determined by the intersection of the region behind the front $\partial_{x} V_{x}^{0}$ and bands (29); in these ranges, we can assume $v_{x \pm} \approx \tilde{v}_{x \pm}$, hence $\partial_{x} v_{x \pm} \approx-\partial_{z} v_{z \pm}$. Thus, $v_{x \pm}$ and $v_{z \pm}$ form an incompressible flow, which is consistent with the property of Alfvén waves to generate only an incompressible flow, which follows from (15). The presence of the compressible part of the $\left(v_{x \pm}, v_{z \pm}\right)$ flow results from non-zero initial conditions (15).

Define also magnetic field perturbations. Once we have found the velocity components, field perturbations can be easily obtained from (5) and (6). Let us use $\tilde{b}_{x \pm}$, $\tilde{b}_{z \pm}$ to denote the asymptotics of perturbations of the $x$ and $z$ components of the nondimensionalized field in Alfvén waves, when $t \rightarrow \infty$. Obtain

$$
\tilde{b}_{x+}=\tilde{v}_{x+} / V_{a}, \tilde{b}_{x-}=-\tilde{v}_{x-} / V_{a},
$$

and

$$
\tilde{b}_{z+}=\tilde{v}_{z+} / V_{a}, \tilde{b}_{z-}=-\tilde{v}_{z-} / V_{a} .
$$

The last two equalities suggest, according to (14, 15), that

$$
\tilde{b}_{z+}=-\frac{1}{2} b^{2}\left(x, z+V_{a} t\right), \tilde{b}_{z-}=-\frac{1}{2} b^{2}\left(x, z-V_{a} t\right) .
$$

Thus, in Alfvén waves far from the source, the external magnetic field always reduces.

\section{CONCLUSION}

It has been shown that in the vicinity of an Alfvén wave its magnetic pressure gives rise to a plasma flow and distorts the magnetic field in a plane perpendicular to the direction of the field perturbation and plasma displacement in such a wave. The second-order compressible perturbation is formed only by the initial perturbation and the interaction between oppositely propagating waves. With distance away from the initial perturbation it becomes negligible and the wave has only the incompressible perturbation, produced by the magnetic pressure of this wave. Thus, at a sufficiently large distance from the region where the initial perturbation occurred, second-order perturbations feature an incompressible plasma flow and field deformation. These perturbations occur in the plane perpendicular to the initial perturbation.

For longitudinal, i.e. directed along the unperturbed magnetic field, plasma velocity and field deformation, there is a very simple relationship between these perturbations and magnetic pressure of the wave, namely: longitudinal velocity and field deformation nondimensionalized by the Alfvén velocity and the unperturbed magnetic field are respectively equal to half the Alfvén wave magnetic pressure nondimensionalized by the magnetic pressure of the external magnetic field. As for the direction of longitudinal perturbations, the longitudinal movement always occurs in the direction of wave propagation, and deformation of the longitudinal field always leads to a reduction of the external field, regardless of propagation direction. If an Alfvén wave is small-scale in a direction perpendicular to the direction of wave propagation, the flow and the field deformation along the direction of small-scaleness are weak, and the flow in the direction of propagation and the trough of the external magnetic field predominate; otherwise, the field perturbations and plasma velocities prevail which are perpendicular to the external magnetic field.

The features of second-order flows identified in this paper allow us to suggest an interpretation of the fast plasma flows observed in the magnetotail as a result of their generation in Alfvén waves. First of all, these are features such as the possibility of the flows to have a direction along the geomagnetic field both to and from Earth. Besides, for the flow velocity $v$ we have $v \sim \varepsilon^{2} V_{a}$, where $\varepsilon$ characterizes the Alfvén wave amplitude; the fulfillment of the condition $\varepsilon^{2}<<1$ is sufficient to apply the results obtained above. Given that the Alfvén velocity in PSBL is as high as $6000 \mathrm{~km} / \mathrm{s}$, as the upper limit of the flow velocity in Alfvén waves within the validity limit we obtain the value of $\sim 1000 \mathrm{~km} / \mathrm{s}$. Thus, generally speaking, we can obtain flows in Alfvén waves throughout the observed range of velocities. Note also that the assumption about the relationship of plasma flows with Alfvén waves do not contradict the observations - the flows are usually accompanied by significant magnetic field perturbations. Separation of observations of the plasma flows driven by Alfvén perturbations and the plasma flows existing independently of Alfvén waves should primarily be based on the following fact. Movement of the region occupied by the plasma flow generated by Alfvén perturbations should 
occur not with the flow velocity, as it should be for the flow existing independently of Alfvén waves, but with the Alfvén velocity. In addition, the velocity of the plasma flow driven by Alfven waves is associated with the Alfvén perturbation amplitude; the magnetic field trough should also be observed.

In connection with the proposed application of the results to the magnetotail plasma sheet boundary layer, it should also be noted that we assume plasma to be uniform, whereas in the said layer it is highly nonuniform in the direction which in notations adopted in this work corresponds to the $x$ coordinate. Given this nonuniformity, the Alfvén velocity in (11) and (12) is a function of $x$. Since the above results indicate that the flow features are determined primarily by local effects of the Alfvén wave magnetic pressure, (11) and (12) with the Alfvén velocity as a function of $x$ can be applied to qualitative estimates with regard for the inhomogeneous structure of PSBL. It is clear that due to the fact that in PSBL the Alfvén velocity increases monotonically along the X-axis (about 10 times on the scale of the order of Earth's radius), the Alfvén perturbation region propagating along the field deforms, extending along the field, but reducing its size in the direction of the inhomogeneity - this means that its transversal scale becomes smaller than the longitudinal one. Hence, whatever the ratio between longitudinal and transversal velocities would be in the flow at the beginning of its formation, with distance the flow becomes onedimensional, directed along the direction of wave propagation, as is mostly observed in fast plasma flows in the magnetotail.

The work was performed with budgetary funding of Basic Research program II.12.

\section{REFERENCES}

Birn J., Liu Y., Daughton W., Hesse M., Schindler K. Reconnection and interchange instability in the near magnetotail. Earth, Planets and Space. 2015, vol. 67, 110. DOI: 10.1186/s40623-015-0282-3.

Cao J.B., Ma Y.D., Parks G., Dandouras H., Remeand I., Nakamura R., Zang T.L., Zong Q., Lucek E., Carr C.M., Liu Z.X., Zhou G.C. Joint observations by Cluster satellites of bursty bulk flows in the magnetotail. J. Geophys. Res.: Space Phys. 2006, vol. 111, iss. A4, A04206. DOI: 10.1029/2005 JA011322.

Dmitrienko I.S. Nonlinear effects in Alfvén resonance. J. Plasma Phys. 1997, vol. 57, no. 2, pp. 311-326.

Dmitrienko I.S. Formation of accelerated ion flows in Alfvén disturbances of the magnetotail // Geomagnetism and Aeronomy. 2011. V. 51, N 8. P. 1160-1164. DOI: 10.1134/ S0016793211080032.

Dmitrienko I.S. Evolution of FMS and Alfvén waves produced by the initial disturbance in the FMS waveguide. J. Plasma Phys. 2013, vol. 79, no. 1, pp. 7-17. DOI: 10.1017/S00 22377812000608.

Du A.M., Nakamura R., Zhang T.L., Panov E.V., Baumjohann W., Luo H., Xu W.Y., Volwerk Q.M., Luand M., Retino A., Zieger B., Angelopoulos V., Glassmeier K.-H., McFadden J.P., Larson D. Fast tailward flows in the plasma sheet boundary layer during a substorm on 9 March 2008: THEMIS observations. J. Geophys. Res.: Space Phys. 2011, vol. 116, iss. A3, A03216. DOI: 10.1029/2010JA015969.

Fruhauff D., Glassmeier K.-H. Statistical analysis of magnetotail fast flows and related magnetic disturbances. Ann. Geophys. 2016, vol. 34, pp. 399-409.
Keiling A. Alfvén waves and their roles in the dynamics of the Earth's magnetotail: a review. Space Sci. Rev. 2009, vol. 142 , iss. $1-4$, pp. 73-156. DOI: $10.1007 / \mathrm{s} 11214-008-$ 9463-8.

Keiling A., Wygant J.R., Cattell C., Temerin M., Mozer F.S., Kletzing C.A., Scudder J., Russell S.T., Lotko W., Streltsov A.V. Large Alfvén wave power in the plasma sheet boundary layer during the expansion phase of substorms. Geophys. Res. Lett. 2000, vol. 27, iss. 19, pp. 3169-3172. DOI: 10.1029/2000 GL000127.

Keiling A., Parks G.K., Wygant J.R., Dombeck J., Mozer F.S., Russell S.T., Streltsov A.V., Lotko W. Some properties of Alfvén waves: observations in the tail lobes and the plasma sheet boundary layer. J. Geophys. Res.: Space Phys. 2005, vol. 110, iss. A10, A10S11. DOI: 10.1029/2004J A010907.

Klimushkin D.Yu., Mager P.N., Pilipenko, V.A. On the ballooning instability of the coupled Alfvén and drift compressional modes. Earth. Planets and Space. 2012, vol. 64, iss. 9, pp. 777-781. DOI: 10.5047/eps.2012.04.002.

Lee D.Y. Ballooning instability in the tail plasma sheet. Geophys. Res. Lett. 1998, vol. 25, iss. 21, pp. 4095-4098. DOI: 10.1029/1998GL900105.

Leonovich A.S., Mishin V.V., Cao J.B. Penetration of magnetosonic waves into the magnetosphere: Influence of a transition layer. Ann. Geophys. 2003, vol. 21, iss. 5, pp. 10831093. DOI: 10.5194/angeo-21-1083-2003.

Leonovich A.S., Kozlov D.A. On balooning instability in current sheets. Plasma Physics and Controlled Fusion. 2013, vol. 55, no. 8, pp. 085013. DOI: 10.1088/07413335/55/8/085013.

Mager P.N., Klimushkin D.Yu. Non-resonant instability of coupled Alfvén and drift compressional modes in magnetospheric plasma. Plasma Physics and Controlled Fusion. 2017, vol. 59, iss. 9, 095005. DOI: 10.1088/1361-6587/aa790c.

Mazur N.G., Chuiko D.A. Kelvin-Helmholtz instability on the magnetopause, magnetohydrodynamic waveguide in the outer magnetosphere, and Alfvén resonance deep in the magnetosphere. Plasma Physics Rep. 2013, vol. 39, no. 6, pp. 488-503.

Mazur N.G., Fedorov E.N., Pilipenko V.A. MHD Waveguides in Space Plasma. Plasma Physics Rep. 2010, vol. 36, no. 7, pp. 609-626. DOI: 10.1134/S1063780X10070081.

Pokhotelov O.A., Onishchenko O.G., Sagdeev R.Z., Treumann R.A. Nonlinear dynamics of inertial Alfvén waves in the upper ionosphere: Parametric generation of electrostatic convective cells. J. Geophys. Res.: Space Phys. 2003, vol. 108, iss. A7, 1291. DOI: 10.1029/2003JA009888.

Pokhotelov O.A., Onishchenko O.G., Sagdeev R.Z., Balikhin M.A., Stenflo L. Parametric interaction of kinetic Alfvén waves with convective cells. J. Geophys. Res.: Space Phys. 2004, vol. 109, iss. A3, A03305. DOI: 10.1029/2003JA010185.

Takada T., Nakamura R., Baumjohann W., Seki K., Voros Z., Asano Z., Volwerk M., Runov A., Zhang T.L., Balogh A., Paschmann G., Torbert R.B., Klecker, B., Reme H., PuhlQuinn P., Canu P., Decreau P.M.E. Alfvén waves in the nearPSBL lobe: Cluster observations. Ann. Geophys. 2006, vol. 24, pp. 1001-1013.

Takada T., Seki K., Hirahara M., Fujimoto M., Hayakawa Y., Saitoand H., Mukai T. Statistical properties of lowfrequency waves and ion beams in the plasma sheet boundary layer: Geotail observations. J. Geophys. Res.: Space Phys. 2005, vol. 110, iss. A2, A02204. DOI: 10.1029/2004JA010395.

Walker A.D.M. Excitation of field line resonances by sources outside the magnetosphere. Ann. Geophys. 2005, vol.

23, no. 1, pp. 3375-3388. DOI: 10.5194/angeo-23-3375-2005.

Wright A.N., Allan W. Simulations of Alfvén waves in the geomagnetic tail and their auroral signatures. J. Geophys. Res. 2008, vol. 113, iss. A2, A02206. DOI: 10.1029/2007JA012464. 
Zhao J.S., Wu D.J., Yu M.Y., Lu J.Y. Convective cell generation by kinetic Alfvén wave turbulence in the auroral ionosphere. Phys. Plasmas. 2012, vol. 19, no. 6, 062901. DOI: 10.1063/1.4729327.

Zong Q.-G., Fu S.Y., Baker D.N., Goldstein M.L., Song P., Slavin J.A., Fritz T.A., Cao J.B., Amm O., Frey H., Korth A., Daly P.W., Reme H., Pedersen A. Earthward flowing plasmoid: Structure and its related ionospheric signature. J. Geophys. Res.: Space Phys. 2007, vol. 112, iss. A7, A07203. DOI: 10.1029/2006JA012112.

How to cite this article

Dmitrienko I.S. Second-order perturbations in Alfvén waves in cold plasma approximation. Solar-Terrestrial Physics. 2019. Vol. 5. Iss. 2. P. 81-87. DOI: 10.12737/stp-52201912. 